\title{
Colossal challenges to healthcare workers combating the second wave of coronavirus disease 2019 (COVID-19) in India
}

\author{
Shayon Ghosh MBBS ${ }^{1}$, Naushin Moledina MBBS${ }^{1}$, Mohammad Mehedi Hasan BSc ${ }^{2}$ (1), Shubhika Jain MBBS $^{3}$ and \\ Asmita Ghosh B.Tech. ${ }^{4}$ \\ ${ }^{1}$ GMERS Medical College and Hospital, Sola, Gujarat, India, ${ }^{2}$ Department of Biochemistry and Molecular Biology, Faculty of Life Science, Mawlana Bhashani \\ Science and Technology University, Tangail, 1902, Bangladesh, ${ }^{3}$ Kasturba Medical College, Manipal, India and ${ }^{4}$ Department of Biotechnology, Heritage \\ Institute of Technology, Kolkata, West Bengal, India
}

To the Editor-Since April 2021, India has been experiencing a massive surge in coronavirus disease 2019 (COVID-19) cases caused by the double mutant or B.1.617 variant. This sudden spike in the number of cases, deemed the second pandemic wave, with a record-breaking $>400,000$ cases daily, has proven to be much more alarming and devastating than the first wave. ${ }^{1}$ The Indian healthcare community is facing major setbacks due to a lack of necessary resources to fight this outbreak, ${ }^{2}$ and healthcare workers (HCWs) are also reaping the consequences. This fight is not only against the virus but also against ignorance. Ignorance and a false sense of normalcy, caused by lack of planning and education, have led to leaders and healthcare institutions being unprepared and people behaving carelessly. ${ }^{3}$

HCWs, along with other frontline warriors, have played a pivotal role in dealing with the pandemic and minimizing the associated hardships. However, India has always had a major imbalance in the number of doctors and healthcare facilities versus the number of people, and the huge population and high doctor-to-patient ratio of $\sim 1: 1,456$, as well as insufficient medical facilities, have added to these hardships. The second pandemic wave has exposed these problems, which usually go unnoticed. Only 8.5 hospital beds and 8 physicians are available per 10,000 population. Apart from the overwhelming number of patients, the precarious lack of manpower and resources is a burden ultimately borne by HCWs. The second pandemic wave has brought higher numbers of moderate-to-severe COVID-19 cases requiring urgent hospital admission. ${ }^{2}$ These sudden increases in hospital admissions and greater demand for medical attention have taken a toll on the well-being of HCWs. Increases in working hours and frequent exposure to severely ill patients have serious repercussions for the health and welfare of doctors, who have been rather neglected in the melee.

Except for a few examples, efforts to analyze and communicate the reality of HCW health conditions have been scarce. The Indian Medical Association (IMA) has cited government data that 87,000 HCWs have been infected and 573 HCWs have died due to COVID-19. Another study by the ICMR has estimated that $5 \%$ of frontline HCWs may have hospital-acquired COVID-19 (HAI) due to the depletion of the facility's workforce. The increasing workload, as well as exposure to patients outside quarantine, create more opportunities for HAI cases. ${ }^{4}$ Another survey has shown that doctors and support staffs are 1.64 times

Author for correspondence: Mohammad Mehedi Hasan, E-mail: mehedi.bmb. mbstu@gmail.com

Cite this article: Ghosh S, et al. (2022). Colossal challenges to healthcare workers combating the second wave of coronavirus disease 2019 (COVID-19) in India. Infection Control \& Hospital Epidemiology, 43: 1302-1303, https://doi.org/10.1017/ice.2021.257 and 5 times more likely to experience pandemic-related burn-out, respectively. Burn-out involves physical, emotional, and mental exhaustion due to long-term involvement in emotionally demanding work, ${ }^{5}$ and it has probably increased the vulnerability of HCWs to various infections in the workplace. The World Health Organization (WHO) recently reported an alarming increase in reports of verbal harassment, discrimination, and physical violence among HCWs due to lack of resources to provide adequate treatment and prevent deaths. ${ }^{6}$ These multifactorial setbacks likely cause a decline in the overall health and performance of HCWs, increase absenteeism, and affect healthcare facilities on a larger scale. In turn, these factors will also contribute to the existing cycle of incompetency in the country's healthcare system.

There is a dire need to attend to these fissures in the healthcare setup to bring a uniform and continuous flow of quality care in India. The WHO has recommended 5 actions to better protect HCWs: (1) protect HCWs from violence; (2) improve HCW mental health; (3) protect HCWs from physical and biological hazards; (4) advance national programs for HCW safety, and (5) connect HCW safety policies to existing patient safety policies. ${ }^{6}$ These steps can be achieved by lowering the exposure of staff by dividing them into teams that work in tandem on alternate days or every third day, based on the number of healthy staff available, inpatient beds, and patient workload. Furthermore, wearing surgical masks and gloves, following hand hygiene protocols, and disinfection of all surfaces between patients in hospitals and clinics should also be mandated. A case report on COVID-19 and the risks to HCWs showed that exposure to aerosol-generating procedures for at least 10 minutes with proper PPE at a $<2 \mathrm{~m}$ from the patient posed low risk of acquiring infection. ${ }^{7}$ As important as these protocols PPE are, their strict implementation should also be exercised regularly.

Above all, the requirement for resources and funding should be undertaken with utmost urgency. No preparedness measures are reliable if hospitals lack basic resources. The government should allocate sufficient funds and build more hospitals and COVID-19 care units to provide sufficient beds for patients with moderate to severe illnesses. ${ }^{2}$ Resources such as ventilators, oxygen cylinders, concentrators, oxygen masks, emergency medications, PPE kits, face shields, N95 masks, and surgical gloves, etc, which are absolute essentials, must be supplied without disruption. Additionally, more health insurance plans and incentives to safeguard the health of HCWs and their families should be initiated. Priority vaccination of not only the healthcare worker but also their immediate family members should be initiated to ensure safety. Along with monetary benefits, a safe workplace environment is 
equally important. Increasing violence and abuse toward HCWs should be taken seriously, and the offenders must be punished to prevent repeated offenses.

A well-planned and specific approach to address healthcare issues is needed, and implementation and follow-up are essential. The COVID-19 pandemic has reminded us all of the importance of HCWs and their role in providing a strong foundation for a country's health and well-being. As the Director-General of WHO, Dr. Tedros Adhanom Ghebreyesus, said, "No country, hospital or clinic can keep its patients safe unless it keeps its health workers safe."

Acknowledgments. None.

Financial support. No financial support was provided relevant to this article.

Conflicts of interest. All authors report no conflicts of interest relevant to this article.

\section{References}

1. India's struggle to track new COVID variants could worsen crisis. Times of India website. https://timesofindia.indiatimes.com/india/indias-struggle- to-track-new-covid-variants-could-worsen-crisis/articleshow/82426964.cms. Accessed May 17, 2021.

2. COVID-19 in India: why the second coronavirus wave is devastating. BBC News website. https://www.bbc.com/news/world-asia-india-56811315.amp. Accessed May 17, 2021.

3. Second wave of COVID-19 to take a toll on Indian healthcare system: Fitch Business-Standard News website. https://www.business-standard.com/article/ current-affairs/second-wave-of-covid-19-to-take-a-toll-on-indian-healthcaresystem-fitch-121041600297_1.html. Accessed May 17, 2021.

4. It's time we took hospital-acquired infections more seriously. The Wire website. https://science.thewire.in/health/its-time-we-took-hospitalacquired-infections-more-seriously/. Accessed May 17, 2021.

5. Khasne RW, Dhakulkar BS, Mahajan HC. Burnout among healthcare workers during the COVID-19 pandemic in India: results of a questionnaire-based survey. Indian J Crit Care Med 2020;24:664-671.

6. Keep health workers safe to keep patients safe. World Health Organization website. https://www.who.int/news/item/17-09-2020-keep-health-workerssafe-to-keep-patients-safe-who. Accessed May 17, 2021.

7. Parikh P, Mehta P, Bansal S, et al. Protecting healthcare professionals and workers (other than COVID-19 management facilities) from contamination during COVID-19 pandemic, March 26, 2020, in India. Indian J Med Sci 2020;72:3-4.

\title{
Influenza vaccination among healthcare personnel during the coronavirus disease 2019 (COVID-19) pandemic
}

\author{
Minji Kang MD ${ }^{1,3}$, Sherry Clark $\mathrm{RN}^{2}$, Sandra Mendoza ${ }^{2}$, Doramarie Arocha $\mathrm{PhD}^{3}$, James B. Cutrell MD ${ }^{1}$, \\ Trish M. Perl MD, MSc ${ }^{1}$ and Julie Trivedi MD ${ }^{1,3}$ \\ ${ }^{1}$ Division of Infectious Diseases and Geographic Medicine, Department of Medicine, University of Texas Southwestern Medical Center, Dallas, Texas, ${ }^{2}$ Department \\ of Occupational Health, University of Texas Southwestern Medical Center, Dallas, Texas and ${ }^{3}$ Infection Prevention, University of Texas Southwestern Medical \\ Center, Dallas, Texas
}

To the Editor-Influenza in healthcare personnel (HCP) may lead to absenteeism and nosocomial outbreaks. ${ }^{1,2}$ The Centers for Disease Control and Prevention, the Advisory Committee on Immunization Practices, and the Healthcare Infection Control Practices Advisory Committee recommend annual immunization of HCP. ${ }^{3}$ During the coronavirus disease 2019 (COVID-19) pandemic, influenza immunization was emphasized vigorously in hope of reducing the burden of respiratory illnesses, medical visits, and hospitalizations that would further strain the healthcare system. We compared influenza vaccine uptake and patterns of declination waivers among HCP before and during the COVID-19 pandemic, and we identified demographics or occupational roles that may be associated with influenza vaccine declination.

At University of Texas Southwestern Medical Center, all HCP are required to complete the annual Seasonal Flu Survey in ReadySet (Axion Health, Broomfield, CO), our employee health software database. On the annual seasonal flu survey, HCP can agree to receive the vaccination at mobile vaccination kiosks or occupational health clinics, upload documentation if vaccinated elsewhere, or decline vaccination by selecting yes or no to 1 or more commonly cited reason for declination. During the 2020-2021

\footnotetext{
Author for correspondence: Minji Kang, E-mail: Minji.kang@utsouthwestern.edu

Cite this article: Kang M, et al. (2022). Influenza vaccination among healthcare personnel during the coronavirus disease 2019 (COVID-19) pandemic. Infection Control \& Hospital Epidemiology, 43: 1303-1305, https://doi.org/10.1017/ice.2021.263
}

influenza season, additional resources such as video series, campus briefings, and town halls were utilized to highlight the importance of influenza vaccination to all HCP. In September, biweekly video series hosted by executive leadership were dedicated to the dual threats of COVID-19 and influenza. The president's biweekly campus briefings highlighted the importance of influenza vaccine, and the emergency operations center sent weekly e-mails to remind HCP of the ongoing influenza campaign.

HCP demographics, occupational role, influenza vaccine uptake, and reasons for declination were obtained for influenza seasons between 2019 and 2021. Data were analyzed using SPSS Statistics version 25 software (SPSS, Armonk, NY). Differences between means were compared using the Student $t$ test, and proportions were compared using $\chi^{2}$ tests. Variables that were statistically significant were analyzed by multivariate logistic regression. All tests were 2 -sided, and $P<.05$ was considered statistically significant. This study was exempt from institutional review board review under category 4 .

Vaccination uptake rate declined significantly during the COVID-19 pandemic, with 13,853 (55.7\%) HCP vaccinated, 1,027 (4.1\%) declined, and 10,010 (40.2\%) with incomplete surveys during the 2020-2021 influenza season compared with 15,757 (63.5\%) vaccinated, 664 (2.7\%) declined, and 8,393 (33.8\%) with incomplete surveys during the 2019-2020 influenza season $(P<.01)$. Safety and/or side effects were the most common reason 